\title{
Analysis of Threshold Current for InP/ AlGaInAs Multiple Quantum Well Ring Laser
}

\author{
Sheng Xie \\ School of Electronic Information Engineer \\ Tianjin University \\ Tianjin, China \\ e-mail: xie_sheng06@tju.edu.cn \\ Hao Wang \\ School of Electronic Information Engineer \\ Tianjin University \\ Tianjin, China
}

\author{
Jing Guo \\ School of Electronic Information Engineer \\ Tianjin University \\ Tianjin, China \\ Weilian Guo \\ School of Electronic Information Engineer \\ Tianjin University \\ Tianjin, China
}

\begin{abstract}
Due to the flexible functions and monolithic integration, semiconductor ring laser (SRL) has become a hot topic in the field of photonic and optoelectronic integration. In this paper, a new threshold current expression suitable for the circular SRL with multiple quantum well (MQW) material structure was derived from the oscillation condition of conventional Fabry-Perot cavity. The influences of ring radius, etching depth and waveguide separation on the threshold current were analyzed and numerically calculated. The calculation results indicated that the total loss of the circular SRL is mainly determined by the ring radius for small-size device. Compared with the shallow-etched devices, the deep-etched SRLs can lase even with smaller radius, and a minimum threshold current of $35 \mathrm{~mA}$ was obtained for InP/ AlGaInAs MQW circular laser.
\end{abstract}

Keywords- semiconductor ring laser; threshold current; multiple quantum well; indium phosphide

\section{INTRODUCTION}

With the rapid development of information society, new communication technologies and applications emerges rapidly, such as high definition video, cloud computing and mobile internet. However, due to the bandwidth mismatch between the optical transmission and the electronic routers, the signal process capability in present optical communication system is increasingly challenged. In order to solve the problem of electronic bottleneck, various implementation schemes have been proposed and employed in optical signal processing, such as semiconductor optical amplifier-based Mach-Zehnder interferometer (SOI-MZI) ${ }^{[1]}$, nonlinear optical loop mirror $(\mathrm{NOLM})^{[2]}$, semiconductor ring laser $(\mathrm{SRL})^{[3]}$, and so on. Compared with other photonic devices, SRLs have attracted more and more attention because they are particularly suited for monolithic integration. SRLs do not require cleaved facets or grating for optical feedback and consume low power. In particular, SRLs can support two counter propagating lasing modes that give rise to a flexible way to process the high-speed optical signal ${ }^{[4]}$. Therefore, SRLs are considered as a potential candidate for constructing large scale photonic integrated circuits.

Up to now, SRLs have been used for tunable laser ${ }^{[5]}$, wavelength-routing switch ${ }^{[6]}$, optical memory ${ }^{[7]}$, and all- optical logic gate ${ }^{[8]}$. More recently, a novel monolithic alloptical toggle flip-flop (TFF) with two complementary output was demonstrated based on the intrinsic bistability of SRL and the saturable absorption effect occurred in the reverse-biased input waveguide, and a recorded bit-rate of $500 \mathrm{Mb} / \mathrm{s}$ for all-optical TFFs was achieved ${ }^{[9]}$.

As a critical parameter of SRLs, the threshold current has importance effects on heat dissipation, laser efficiency, and mode stability. To reduce the threshold current of SRLs, several approaches, such as optimizing the device geometry and improving the fabrication process, have been employed.

In this paper, a threshold current expression, which suitable for the case of circular SRL with two coupled straight waveguides, was derived from the oscillation condition of Fabry-Perot cavity. The influences of key device physical parameters, such as ring radius, etching depth, and waveguide separation, on the threshold current were analyzed and numerically calculated.

\section{THEORETICAL ANALYSIS}

We consider a SRL like the one schematically depicted in Figure 1. It consists of a ring waveguide cavity with radius $\mathrm{R}$ and two output straight waveguides that are coupled to the ring cavity via directional coupler. To improve the coupling efficient between the output waveguide and the optical fiber, a taper structure terminating the output waveguide was employed. The

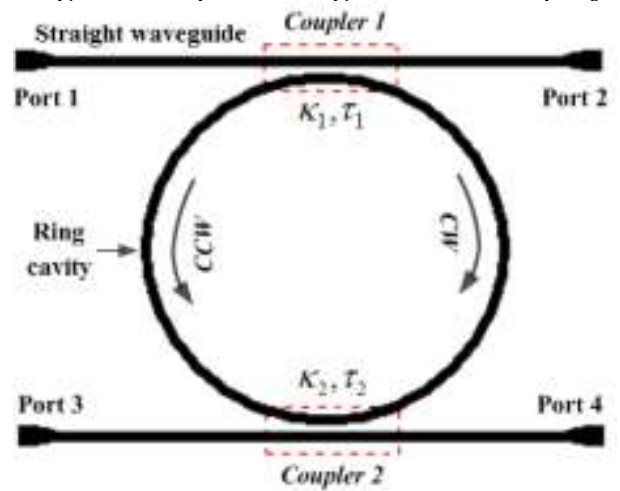

Figure 1. Schematic illustration of a circular SRL with two coupled straight waveguides 
amplitude coupling ratio and the amplitude transmission ratio of the couplers are denoted as $\kappa$ and $\tau$, respectively. We suppose the SRL is fabricated by InP/AlGaInAs MQW structure, where the MQW active region is sandwiched by graded-index separate confinement heterostructure (GRIN$\mathrm{SCH}$ ), and the upper and lower claddings are InP material.

Figure 2 shows the cross-section of the MQW ridge waveguide. Where $W$ is the ridge width, $d$ is the etching depth from the top of GRIN-SCH, $\mathrm{n}_{1}$ represents the air index, $n_{2}$ and $n_{3}$ are the indices of the cladding and the core layer, respectively.

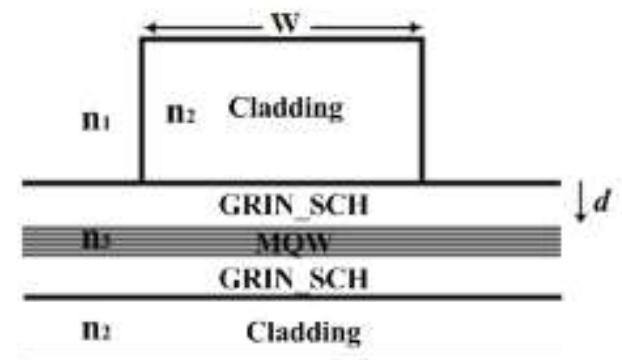

Figure 2. Cross-section of the MWQ ridge waveguide

Based on the laser oscillation condition of a FabryPerot cavity ${ }^{[10]}$, the threshold gain should be modified to be adapted to the SRL. So the formula for the circular SRL as shown in Figure 1 can be expressed as

$$
T_{1} T_{2} \exp [(g-\alpha) L] \exp \left[\frac{i 2 \pi n_{e f f} L}{\lambda}\right]=1
$$

Where $T_{1,2}=\tau_{1,2}^{2}=1-\kappa_{1,2}^{2}$ are, respectively, the power transmission ratios of the coupler 1 and coupler $2, g$ is the material gain, $\alpha=\alpha_{i}+\alpha_{b}+\alpha_{s c}$ is the loss of ring cavity, $\alpha_{i}$ is the intrinsic waveguide loss, $\alpha_{b}$ represents the bending loss, which is a function of the radius and the etching depth, $\alpha_{s c}$ represents the scattering loss caused by ridge waveguide sidewall roughness, $L=2 \pi R$ is the cavity length, $R$ is the ring radius, $n_{\text {eff }}$ is the effective index of ring cavity, $\lambda$ is the wavelength.

From equation (1), the resonance condition and the gain condition of a circular SRL can be written as

$$
\begin{aligned}
& m \lambda=n_{\text {eff }} \cdot L \\
& T_{1} T_{2} \exp [(g-\alpha) L]=1
\end{aligned}
$$

Where $m$ is a positive integer. The wavelength $\lambda$, which satisfies the oscillation condition, will be selected and lased. The lasing light will subsequently couple evanescently to the straight waveguide and guide to the output port.

Laser oscillation takes place when the optical gain is equal to the total loss, which is the sum of the loss of the ring cavity and the coupling loss. Therefore, the threshold gain extracted from equation (3) can be expressed as following

$$
g_{\text {th }}=\alpha+\frac{1}{L} \ln \left(\frac{1}{T_{1} T_{2}}\right)=\alpha+\alpha_{c}
$$

Where $\alpha_{c}$ represents the coupling loss.

Based on the coupling-mode theory, the amplitude coupling ratio $\kappa$ of the coupler shown in Figure 1 can be expressed as ${ }^{[11]}$

$$
\kappa=\sin \left[R \int_{-\pi / 2}^{\pi / 2} K_{/ /}(\theta) \cos ^{2} \theta d \theta\right]
$$

Where $K_{/ /}(\theta)$ represents the coupling coefficient between two coupled waveguides with tilt angle of $\theta$, and the exact values can be obtained by solving the characteristic equations of specific waveguide geometry.

The bending loss of ring waveguide is given by ${ }^{[12]}$

$\alpha_{b}=\frac{n_{1}^{2}}{n_{3}^{2}}\left[1-\frac{n_{1}^{2}}{n_{3}^{2}}\right]^{-\frac{1}{2}} \exp \left\{-\frac{\Re}{3}\left[1-\left(\frac{9 \pi}{2 \mathfrak{R}}\right)^{\frac{2}{3}}+\frac{4 n_{1}^{2}}{n_{3}^{2} \mathfrak{R}}\right]^{\frac{3}{2}}\right\}$

Where $\mathfrak{R}=2 \pi^{2} R\left(n_{3}-n_{2}\right)^{3 / 2} / k_{x 0}^{2} \lambda^{3}, \quad k_{x 0}$ represents the propagation constant in the $x$ direction, and can be obtained by solving the characteristic equations.

For the case of narrow or strained MQW laser, the relation of gain and current density can be approximated as $^{[13]}$

$$
g=g_{0}\left[1+\ln \left(\frac{J}{J_{0}}\right)\right]
$$

Where $J$ is the current density injected into the active region, $J_{0}$ and $g_{0}$ are, respectively, the current density coefficient and gain coefficient, the modal material gain is given by $g_{m}=n_{w} \Gamma_{w} g, n_{w}$ is the number of quantum wells, $\Gamma_{w}$ is the optical confinement factor of one well, $g$ is the material gain per quantum well.

If the amplitude coupling ratio $\kappa$ of both couplers is same, $\kappa_{1}=\kappa_{2}=\kappa$, then the threshold current of MQW circular SRL can be expressed as

$$
\begin{aligned}
I_{t h} & =J_{0} W L \exp \left(\frac{\alpha_{i}+\alpha_{b}+\alpha_{s c}-2 \operatorname{In}\left(1-\kappa^{2}\right) / L}{n_{w} \Gamma_{w} g_{0}}-1\right) \\
& =J_{0} W L \exp \left(\frac{\alpha-2 \operatorname{In}(T) / L}{n_{w} \Gamma_{w} g_{0}}-1\right)
\end{aligned}
$$

\section{RESULTS AND DISCUSSION}

To ensure effectively single mode behavior as well as ease of fabrication, the ridge width $W$ around $2 \mu \mathrm{m}$ for the practically fabricated SRL was generally selected ${ }^{[14,15]}$, so $W=2 \mu \mathrm{m}$ is adopted in the following simulation. Because the bending loss $\alpha_{b}$ is a strong function of ring radius and etching depth, as described in equation (6), two values for the etching depth, namely $\mathrm{d}=0 \mathrm{~nm}$, and $\mathrm{d}=60 \mathrm{~nm}$, are considered for varying radius. Since the scattering loss $\alpha_{s c}$ increases with the increase of sidewall roughness and etching depth, we estimate that $\alpha_{s c}=20 \mathrm{~cm}^{-1}$ for $\mathrm{d}=0 \mathrm{~nm}$, while $\alpha_{s c}=60 \mathrm{~cm}^{-1}$ for $\mathrm{d}=60 \mathrm{~nm}^{[13]}$. Other physical parameters adopted in the simulation are summarized in Table 1.

The power transmission ratio $T$ of the circular SRL depends strongly on the separation between the straight and ring waveguide and the ring radius. Numerical calculations using optical simulation software were carried out to investigate the influence of these parameters on the power transmission ratio. Figure 3 shows the calculation results for $\mathrm{d}=0 \mathrm{~nm}$. Initially, the power transmission ratio increases exponentially with increasing the waveguide separation, but it is close to unity when the separation 
TABLE 1 PHYSICAL PARAMETERS USED IN THE SIMULATION ${ }^{[13,16]}$

\begin{tabular}{cll}
\hline Symbol & \multicolumn{1}{c}{ Description } & \multicolumn{1}{c}{ Value } \\
\hline$n_{l}$ & Index of air & 1.0 \\
$n_{2}$ & Index of core & 3.5 \\
$n_{3}$ & Index of cladding & 3.17 \\
$\alpha_{i}$ & Intrinsic waveguide loss & $20 \mathrm{~cm}^{-1}$ \\
$n_{w}$ & Number of quantum wells & 5 \\
$\Gamma_{w}$ & Confinement factor per well & 0.02 \\
$\lambda$ & Photon wavelength & $1550 \mathrm{~nm}$ \\
$g_{0}$ & gain coefficient & $600 \mathrm{~cm}^{-1}$ \\
$J_{0}$ & Current density coefficient & $1700 \mathrm{~A} / \mathrm{cm}^{2}$ \\
\hline
\end{tabular}

exceeds $1.0 \mu \mathrm{m}$. For a given waveguide separation, due to the stronger coupling ratio for larger radius, the power transmission ratio decreases with the increase of radius.

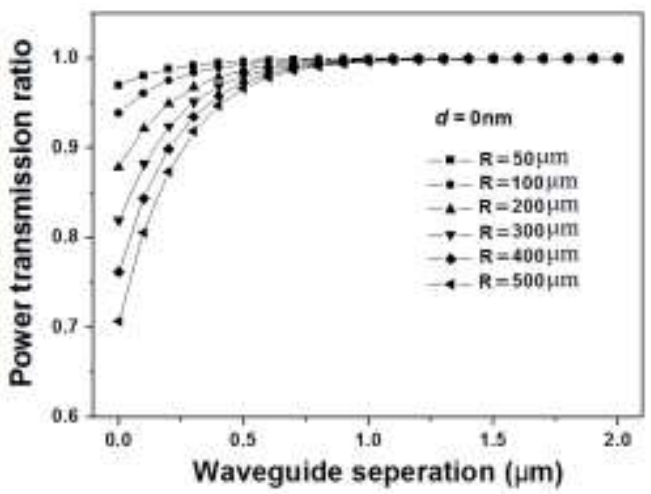

Figure 3. The power transmission ratio as a function of waveguide separation for different radii

The calculated bending loss as a function of radius is plotted in Figure 4. It is shown that the bending loss decreases rapidly with increasing the ring radius, and tends to the asymptotic value of zero for large ring radius. For the case of etching depth $d=0 \mathrm{~nm}$ the bending loss become very high for radii smaller than $100 \mu \mathrm{m}$, while for $d=60 \mathrm{~nm}$ this happens for radii smaller than $50 \mu \mathrm{m}$. Therefore, small-radii circular SRLs are only practicable for deep-etched device. Unfortunately, deep etching brings about additional scattering loss due to the ridge sidewall roughness, and in some cases it determines the total cavity loss. As results, an appropriate etching depth needs to be selected to obtain the smallest loss.

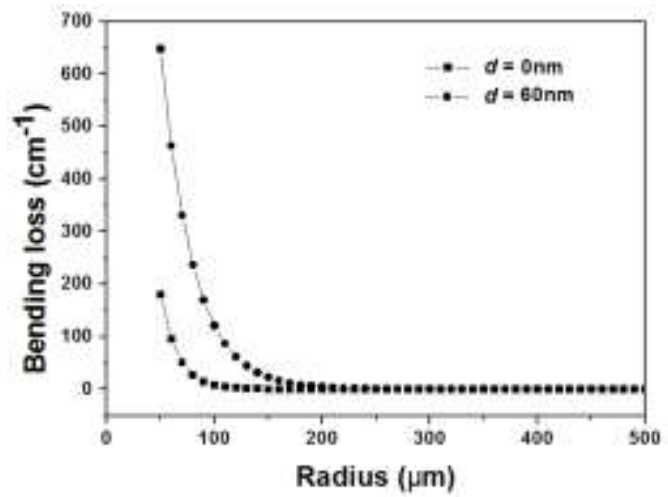

Figure 4. Simulated bending loss as a function of SRL radius for different etching depths.
Figure 5 shows the coupling loss versus the radius for two values of the waveguide separation. Compare to the total cavity loss, the coupling loss is so smaller that it can be ignored in calculating the threshold current. This is a typical feature of the circular SRLs.

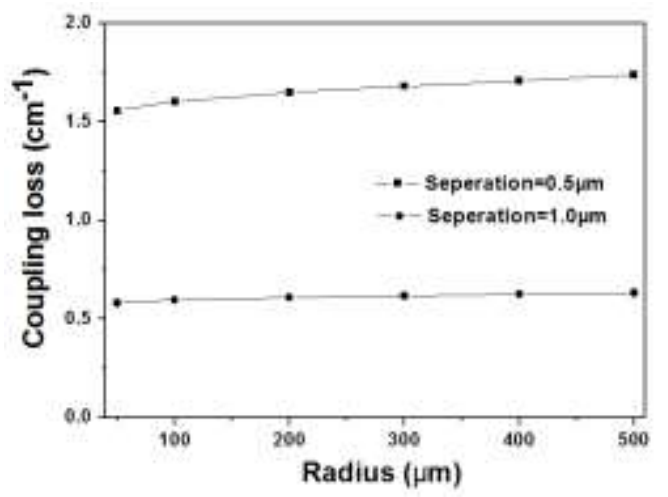

Figure 5. Simulated coupling loss versus radius for different waveguide separations

Based on the calculation results of power transmission ratio, bending loss, and coupling loss, the threshold current $I_{t h}$ can be calculated using equation (8), and the results are plotted in Figure 6. As can be seen from this figure, initially, the threshold current decreased rapidly with increasing SRL radius due to the decreased bending loss, and a minimum value is obtained. However, the threshold current goes up for large SRL radius, this is caused by the increased scattering loss and intrinsic waveguide loss. A minimum threshold current $31 \mathrm{~mA}$ can be achieved for a shallow-etched circular SRL with radius of $170 \mu \mathrm{m}$ However, Due to the increased scattering loss with increasing the etching depth, the minimum threshold current increases slightly, and it value equals $34 \mathrm{~mA}$ for a deep-etched circular SRL with a corresponding ring radius of $100 \mu \mathrm{m}$.

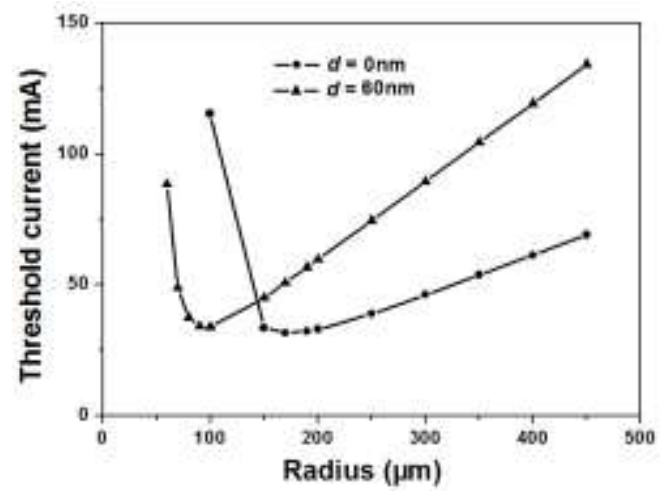

Figure 6. Simulated threshold currents as a function of SRL radius for different etching depths.

\section{CONCLUSION}

A threshold current expression suitable for the semiconductor ring laser with circular geometry was derived from the oscillation condition of conventional Fabry-Perot cavity. The influences of ring radius, etching depth and waveguide separation on the threshold current were analyzed and numerically calculated. The calculation results indicated that the total loss of the circular SRL is mainly determined by the ring radius for small-size device, 
because the bending loss has a strong dependence on the radius. Compared with the shallow-etched device, the deep-etched SRL can lase even with smaller ring radius, and a minimum threshold current of $35 \mathrm{~mA}$ is obtained using the expression derived from Fabry-Perot cavity. To achieve high density integration with small-size devices in the future, the loss of ring cavity needs to reduce further. This can be done either by choosing a deep-etched circular geometry with optimized inductive couple plasma (ICP) etching parameters, or by reverting to the square geometry with shallow-etched waveguide.

\section{ACKNOWLEDGMENT}

The work was supported by the National Natural Science Foundation of China under Grant 61106052.

\section{REFERENCES}

[1] D. K. Gayen, A. Bhattachryya, T. Chattopadhyay, and J. N. Roy, "Ultrafast all-optical half adder using quantum-dot semiconductor optical amplifier-based Mach-Zehnder interferometer," J. Lightw. Technol., vol.30, pp. 3387-3393, Nov. 2012.

[2] D. Williams, X. Bao, and L. Chen, "All-optical NAND/NOT/AND /OR logic gates based on combined brillouin gain and loss in an optical fiber," Appl. Opt., vol.52, pp. 3404-3411, May, 2013.

[3] S. Y. Yu, "All-optical functions based on semiconductor ring lasers," Chin. Opti. Lett., vol.8, pp. 918- 923, Sep. 2010.

[4] M. F. Booth, A. Schremer, and J. M. Ballantye, "Spatial beam switch and bistability in a diode ring laser," APPL. PHYS. LETT., vol.76, pp. 1095-1097, Feb, 2000.

[5] S. Kim, Y. T. Byun, D. G. Kim, N. Dagli, and Y C Chung, "Widely tunable coupled ring reflector laser diode consisting of square ring resonators," J. Opt. Soc. Korea, vol.14, pp. 38-41, Mar. 2010 .
[6] T. Segawa, S. Matsuo, T. Kakisuka, Y Shibata, and T. Sato, "Monolithically integrated wavelength-routing switch using tunable wavelength converters with double-ring-resonator tunable Lasers," IEICE Trans. Electron., vol.E94-C, pp. 1439-1446, Sep. 2011.

[7] M. T. Hill, H. J. S. Dorren, T. de Vries, J. M. Leijtens, and J. H den Besten, "A fast low-power optical memory based on coupled micro-ring laser”. Nature, vol.432, pp. 206-208, Nov. 2004.

[8] B. Li, M. I. Memon, G. Mezosi, Z. Wang, M. Sorel, and S Yu, "All-optical digital logic gates using bistable semiconductor ring lasers," J Opt. Commun., vol.30, pp.190-194, Dec. 2009.

[9] A. Trita, G. Mezosi, M. Sorel, G. Giuliani, “All-optical toggle flipflop based on monolithic semiconductor ring laser". IEEE Photon. Technol. Lett. vol.26, pp. 96-99, Jan. 2014.

[10] J. P. Jiang. Semiconductor laser, Beijing: Publishing House of Electronic Industry, 2000, pp.52-57.

[11] C. S. Ma, and S. Y. Liu, Mode theory of optical waveguide, Changchun: Jinlin University Press, 2007, pp.339-347.

[12] H. B. Chen, Y. Q. Huang, H Huang, Y. F Xu, A. Miao, Y. Q. Li, and X. M. Ren, "Analysis of output coupling coefficent and threshold of semiconductor microring laser," J. Optoelctron. Laser, vol.18, pp.2013-2132, May 2007.

[13] G Giuliani, A Scire, M Sorel, S. Donati, "Linewidth of monolithic semiconductor ring lasers," Proceeding of SPIE, 6184, Semiconductor Lasers and Laser Dynamics II, April 2006, pp. 640647.

[14] G. Mezösi, M. J. Syrain, S. Fürst, Z. Wang, S Yu, and M. Sorel, "Unidirectional bistability in AlGaInAs microring and microdisk semiconductor lasers," IEEE Photon. Technol. Lett., vol.21, pp.8890, Jan. 2009.

[15] S. Wang, Y. Huang, Y. Yang, J. Lin, K. Chen, J. Xiao, and Y Du, "Long rectangle resonator 1550nm AlGaInAs/InP lasers," J. Opt. Soc. Am. B, vol.27, pp. 719-724, April 2010.

[16] G. Mezosi, "Semiconductor ring lasers for all-optical signal processing," Glasgow: Univertiy of Glasgow, 2010, pp. 35-41. 